\title{
Recognition of online unconstrained handwritten Gurmukhi characters based on Finite State Automata
}

\author{
HARJEET SINGH*, R K SHARMA and V P SINGH \\ Department of Computer Science and Engineering, Thapar Institute of Engineering \& Technology (Deemed \\ University), Patiala, India \\ e-mail: harjeet.singh@thapar.edu; rksharma@thapar.edu; vpsingh@thapar.edu
}

MS received 24 March 2017; revised 24 September 2017; accepted 4 May 2018; published online 23 October 2018

\begin{abstract}
This paper presents a language-based efficient post-processing algorithm for the recognition of online unconstrained handwritten Gurmukhi characters. A total of 93 stroke classes have been identified to recognize the Gurmukhi character set in this work. Support Vector Machine (SVM) classifier has been employed for stroke classification. The main objective of this paper is to improve the character level recognition accuracy using an efficient Finite State Automata (FSA)-based formation of Gurmukhi characters algorithm. A database of 21,945 online handwritten Gurmukhi words is primarily collected in this experiment. After analysing the collected database, we have observed that a character can be written using one or more strokes. Therefore, a total of 65,946 strokes have been annotated using the 93 identified stroke classes. Among these strokes, 15,069 stroke samples are considered for training the classifier. The proposed system achieved promising recognition accuracy of $97.3 \%$ for Gurmukhi characters, when tested with a new database of 8,200 characters, written by 20 different writers.
\end{abstract}

Keywords. Online handwriting recognition; Gurmukhi characters; post-processing; strokes association; Finite State Automata; SVM classifier.

\section{Introduction}

Study in the field of online handwriting recognition for Indian scripts has become popular nowadays. More attention is given towards online handwriting recognition because of these reasons: (i) handwriting is easier than typing, (ii) typing is not possible everywhere, (iii) nonavailability of all languages keyboard and (iv) typing is difficult for varied size of characters. Moreover, due to rapid revolution in devices related to Information Technology, including mobiles based on touch screens, digital tablets, notepads, etc., the demand of applications based on online handwriting recognition is increasing day by day. These devices help us in capturing information with the help of a digital-pen/stylus. This captured information is stored as $x-y$ traces with progressive time. A set of such traces is referred to as a stroke in an online handwriting recognition system. A stroke is thus the set of coordinates captured between the two events, namely, pen-down and pen-up with the help of a writing device. A stroke is the building block in online handwriting recognition systems.

Online handwritten character recognition of any scripting language is a difficult task due to the problems of different handwriting styles of different individuals, complex

*For correspondence structure of a language and different touch-based handwriting capturing devices used by the individuals. A good amount of research work has been carried out on online handwriting recognition in the recent past for different scripts such as Chinese, Japanese, Korean and Arabic [1-4]. On the other hand, works on isolated character recognition for many Indic languages like Bangla, Hindi, Tamil and Telugu have been reported by many researchers in the past few years [5-8]. The present study deals with recognition of Gurmukhi script, the most renowned script of north India used to write the Punjabi language. A stroke is considered as the smallest unit in online Gurmukhi script recognition. Further, a Gurmukhi character can be formed using a single recognized stroke or with a combination of more than one recognized stroke. The most common difficulties that occur in the online handwritten Gurmukhi script recognition are

- complex structure of the script,

- similar shape of characters,

- huge variation in handwriting styles,

- formation of a character by combining the strokes, etc.

The post-processing phase of online handwriting recognition system deals with character formation, which plays a crucial role in order to improve the accuracy of the recognition system. In the literature, the researchers have 
mostly used the rule-based approach to form a character from the recognized strokes [9-12]. Rule-based methods have certain limitations such as the following: (i) not giving the desired output in case the input data are insufficient and (ii) difficult to write the generic rules to deal with all the situations. However, in the present work, a Gurmukhi character is formed using a different approach, based on Finite State Automata (FSA) theory, which covers all the possible variations used to write a Gurmukhi character and overcome the limitations of rule-based approach. We here present two algorithms, one is an efficient algorithm that reorders the lists of recognized strokes in such a way so as to render formed character(s) in a valid sequence. Another one is FSA-based Gurmukhi character formation algorithm, which forms a character from the sequence of strokes available in the lists.

The rest of the paper is organized as follows. In section 2 , we present a brief review of the related works. A brief discussion about the Gurmukhi script is given in section 3. In section 4, various challenges to Gurmukhi character are discussed. Section 5 describes about the online handwritten data collection. The pre-processing steps and features used in the present study are discussed in section 6 . The classification tool used for stroke classification has been discussed in section 7. Section 8 elaborates the detailed process of Gurmukhi character formation. Experimental results are presented in section 9 and concluding remarks are given in section 10.

\section{Related work}

This section briefly reviews the literature on online handwriting recognition systems. Tappert et al [13] have described the state of art in online handwritten character and word recognition systems. Shape recognition algorithms, pre-processing techniques and post-processing techniques have extensively been surveyed in their paper. Gao et al [14] presented an online handwritten Japanese text recognition system. Their system includes an offline character recognizer and other components for linguistic and geometric contexts. A linear Markov Random Field (MRF) chain model has been used in their work. They have reported an accuracy varying from $91.1 \%$ to $95.7 \%$ by combined the recognizer. Aparna et al [15] presented a recognition system for online handwritten Tamil characters. In their work, a set of 18 shape features is used for stroke recognition and they implemented the character formation scheme using FSA theory. They employed an accuracy of $82.80 \%$ for Tamil characters. Swethalakshmi et al [16] developed an online handwritten character recognition system for Devanagari and Telugu scripts. The Support Vector Machine (SVM) classifier has been used for stroke recognition. In post-processing, rule-based strokes database is used for character formation in their work. Rampalli and
Ramakrishnan [17] have proposed an online handwritten character recognition system working in blend with an offline recognition system for Kannada. In their work, online written strokes are converted into an offline image and concurrently recognized by both online and offline strategies. They have reported an improvement of $11.0 \%$ in accuracy by combining the online recognition system with offline one. Roy et al [18] have discussed online handwritten Bangla characters formation. In their study, they have computed a total of 15 structural features and 90 point-based features. The Multilayer Perceptron Neural Network scheme has been used for stroke recognition. They have followed rule-based strategy for Bangla character formation. Later on, Samanta et al [19] proposed a novel approach for online unconstrained handwritten Bangla words recognition using the hidden Markov (HMM) model. In their work, a whole word sample is considered as a basic unit for recognition instead of recognizing individual substrokes. Circular feature, linear feature and combination of circular and linear features have been computed at substrokes level after segmenting the word into sub-strokes. They have implemented fully connected non-homogeneous HMMs in their experiments. The applied smoothing of the probability estimates at two levels shows the promising results in online handwritten Bangla word recognition.

A very limited amount of research work has been carried out on online handwritten character recognition for Gurmukhi script in recent past. Initially, the work in online handwritten Gurmukhi script was started with basic characters recognition using elastic matching technique [20]. In this study, online Gurmukhi character is formed using a single or more than one stroke and the strokes are preprocessed through five steps (i.e., normalization and centring, interpolating missing points, smoothing, slant correction and re-sampling of points) in sequential order. Later, Sharma et al [21] extended this work by introducing a new approach of rearrangement of recognized strokes on the basis of dependent and major dependent strokes before recognition of a particular Gurmukhi word. Afterwards, in [22], the 41 Gurmukhi characters recognition was studied using a HMM-based classifier. In 2013, Kumar and Sharma [10] presented an efficient algorithm for post-processing of online handwritten Gurmukhi character recognition where the characters were formed by a rule-based method. Later on, Kumar et al [11] discussed the recognition of multistroke-based online handwritten Gurmukhi Aksharas, where SVM classifier has been used for stroke recognition and rule-based approach was used for character and Aksharas formation. In their work, they have tested 4,310 Gurmukhi Aksharas, written by 10 different writers. They have also shown the results of 35 Gurmukhi characters with a combination of Vowel and Nasal. In [23], online handwriting recognition procedure for Gurmukhi script was discussed. Various challenges, like zone identification of a stroke, identification of location of headline and dot detection, were discussed in pre-processing phase. 
Moreover, in classification phase, rule-based methods and statistical classification methods like TDNN, HMM and SVM were also discussed. In a recent study [24] of online handwritten Gurmukhi character recognition, a votingbased classification model is implemented based on HMM and SVM classifiers. In their work a total of 74 stroke classes have been identified in order to implement Gurmukhi character recognition. Stroke classification has been carried-out based on five different features, namely, normalized $x-y$ traces $\left(N_{x y}\right)$, region-based features $\left(R_{x y}\right)$, curvature features $\left(C_{x y}\right)$, curvature-feature-based classes $\left(C_{x y}^{N}\right)$ and directional features $\left(D_{x y}\right)$. In post-processing, they have used the rule-based method proposed by Kumar and Sharma [10] for character formation from the recognized strokes. In experimentation, they have considered a test data set of 35 Gurmukhi consonants, which was written by 10 different writers, five times each. Additionally, the major issues of confusing characters formation have also been studied by them.

\section{Gurmukhi script}

Gurmukhi is the script used for writing Punjabi language. Punjabi is an Indo-Aryan language spoken by 102 million speakers worldwide. It is the $10^{\text {th }}$ most widely spoken language in the world. In the $16^{\text {th }}$ century, Shri Guru Angad Dev Ji, second Sikh Guru, standardized the Gurmukhi

\begin{tabular}{|c|c|c|c|c|c|c|c|c|c|c|c|}
\hline \multirow{4}{*}{ 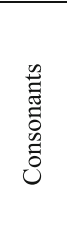 } & 8 & भr & प्ट & म & J & व & ษ & गा & щ & қ & \\
\hline & $\nabla$ & ह & त & ₹ & हृ & ट & ঠ & る & छ & $\Sigma$ & \\
\hline & 3 & घ & 己 & प & চ & ૫ & ढ & घ & ब & भ & \\
\hline & ज & ஏ & ম & ट & इ & म & 부 & गा & त़ & ढढ & पू \\
\hline $\begin{array}{l}\frac{n}{0} \\
0 \\
0 \\
0 \\
>\end{array}$ & $\mathrm{j}^{\mathrm{T}}$ & fo & ओी & 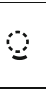 & 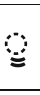 & $\theta$ & 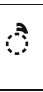 & $\Leftrightarrow$ & a & & \\
\hline 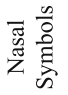 & $a^{n}$ & $\because$ & 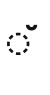 & & & & & & & & \\
\hline
\end{tabular}

Figure 1. Gurmukhi character set.

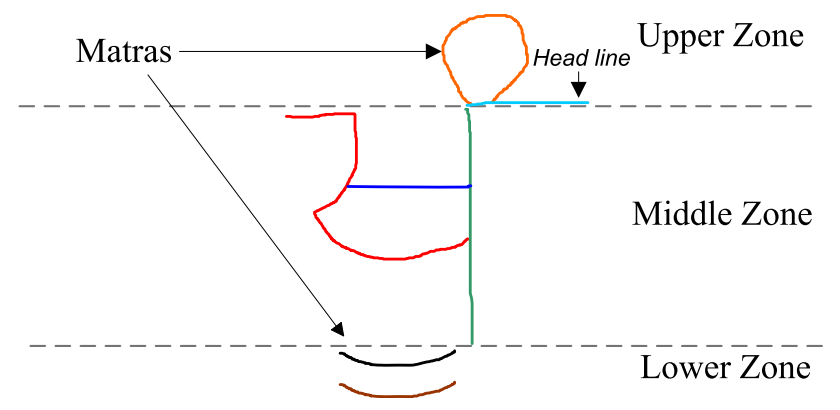

Figure 2. Online handwritten Gurmukhi script representation in three zones. Different colours indicate different strokes within a character sample. script. The name of Gurmukhi is derived from the old Punjabi term Guramukhi, which means from the mouth of Guru. The following are some of the basic characteristics of Gurmukhi script: (i) the writing structure of Gurmukhi script is cursive and (ii) it is written from left to right direction. Figure 1 illustrates various characters of the Gurmukhi script. It has a basic set of 53 characters: 41 consonants ( 35 basic consonants +6 additional modified consonants (i.e., म, Ұ, गा, न., ढ, and ए़), 9 vowels and 3 nasal symbols. Among these 9 vowels, 4 vowels (i.e.,,$\vec{\partial}$ and $\curvearrowright$, considered as upper matras, reside above the consonants; 2 vowels (i.e., 9 and $\cong$ ), considered as lower matras, reside below the consonants and 3 vowels (i.e., r, f and a) appear adjacent to a consonant. The nasal symbols (i.e., $\dot{0}, \dot{0}$ ) are also considered as upper matras and they also reside above the consonants [25].

\subsection{Set of strokes considered in this work}

Online handwritten strokes for Gurmukhi script can be drawn in one of the three horizontal zones, namely, Upper Zone, Middle Zone and Lower Zone as shown in figure 2. In Gurmukhi script the consonants are formed along with a horizontal line, called head-line, drawn above the consonant. Upper matras strokes can be separated on the basis of this head-line. To detect this head-line is the major problem in Gurmukhi character recognition. Samanta et al [19] proposed an algorithm to detect the headline and baseline to classify the strokes into three zones. In the present work, the same scheme is used to detect the headline, which helps classify the strokes into two zones (i.e., Upper Zone and MiddleLower Zone).

To identity the stroke classes for online handwritten Gurmukhi character recognition system, we analysed the collected handwritten data. During analysis, we identified the shapes of strokes, used by different writers, to write a Gurmukhi character. In this way, all the possible variations for writing the Gurmukhi characters have been observed. This resulted in a total of 93 stroke classes. Among these identified stroke classes, there exist some strokes that are similar in shape and participate in the formation of consonants and vowels, leading to a confusion to the recognizer, if classified using a single classifier. In order to reduce this confusion between the stroke classes and increase stroke-level classification accuracy, these stroke classes are further decomposed into two subsets: (i) Upper Zone stroke set and (ii) MiddleLower Zone stroke set, shown in figure 3. The Upper Zone stroke set contains 12 stroke classes to classify the upper matras and the MiddleLower Zone stroke set contains 81 stroke classes to classify all the consonants and lower matras. Therefore, two zone-wise classifiers, namely, UpperZoneClassifier and MiddleLowerZoneClassifier, are trained to classify the input strokes in the respective 
zones. In the present study, the reasons to keep the stroke classes of lower matras and consonants in a single set are the following: (i) the count of lower matras is less in comparison with consonants (i.e., lower matras $=2$, and consonants $=41$ ) and (ii) the stroke classes lower matras

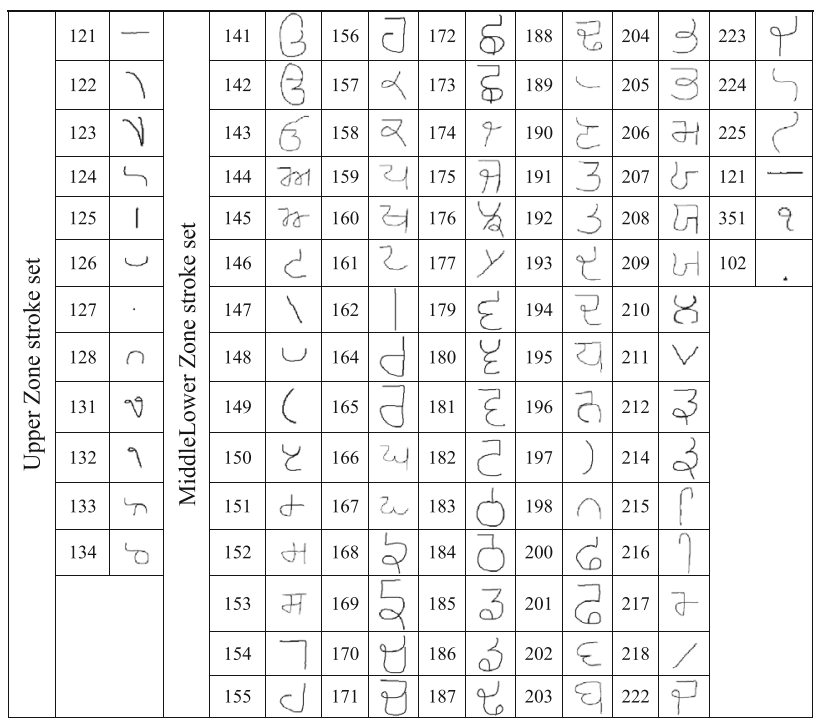

Figure 3. Shapes of strokes considered for Gurmukhi character recognition system. The three digits numerical values represent stroke labels assigned to the respective stroke shapes.

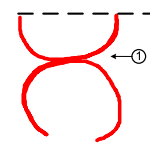

(a)

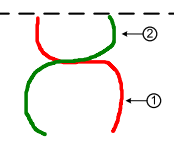

(b)

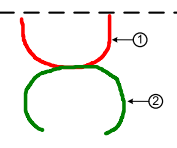

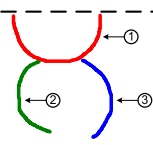

(d)

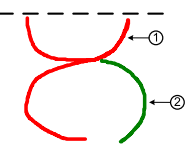

Figure 4. Variations in writing Gurmukhi character '"ॅ': (a) the character ' ' is written in a single stroke, (b) written in two strokes, (c) written in two strokes, but with different shapes, (d) written in three strokes and with different shapes and (e) written in two strokes and again with different shapes. (For interpretation of the references to colour in this figure, the reader is referred to the web version of this paper).

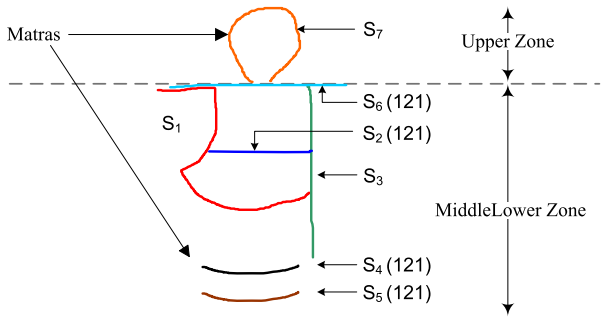

(a) are confused with the stroke classes of consonants. Therefore, the stroke classes to classify the lower matras $(;$, and $)$ are separated from MiddleLower Zone on the basis of their position on $y$-axis in post-processing phase.

\section{Challenges in online handwritten characters formation}

Due to variation in handwriting, writers have their own way of writing, which poses a major challenge to character recognition. Writers write characters in different sizes. It has also been observed that a Gurmukhi character can be formed using different combinations of strokes, as illustrated in figure 4. This makes the character formation difficult.

Moreover, when a character is formed by combining the predicted online input strokes, the problem of strokes merging, strokes discarding and strokes sequencing arises, where it may be possible that online input strokes belong to same character but their sequence of input may be different, so these types of issues are handled by stroke merging strategy.

Apart from this, a problem of ambiguity between two or more strokes that may arise due to similar features of strokes that belong to different Unicode characters is illustrated in figure 5 . It has been resolved by identifying the position of ambiguous strokes on $x$-axis and $y$-axis (horizontal and vertical projection, respectively) and then their assigned stroke labels are changed accordingly. In figure 5a, four strokes, $s_{2}, s_{4}, s_{5}$ and $s_{6}$, are of similar shape. The predicted stroke label of these strokes is "121", associated with stroke sequence number. This ambiguity has been resolved by replacing the stroke label of strokes $s_{2}, s_{4}$ and $s_{5}$ with "163", "101" and "101", respectively, shown in figure $5 \mathrm{~b}$. Stroke classes for lower matras (i.e.,,$\bigcirc$ and are then extracted from MiddleLower Zone.

In the literature, many researchers have used the rulebased approach to combine the recognized strokes

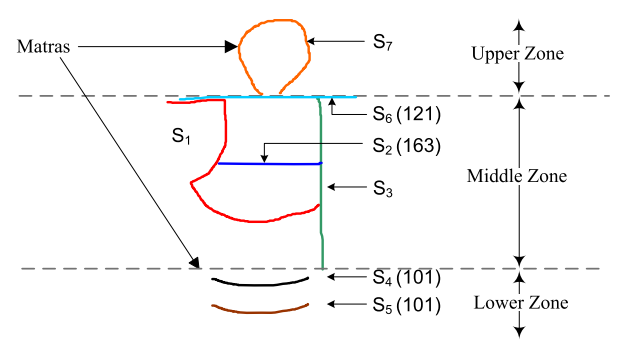

(b)

Figure 5. Similar features of strokes in online handwritten Gurmukhi word "स्झ". In (a), the stroke label of the lower matras strokes $s_{4}$ and $s_{5}$ is " 121 ", which is replaced with the new stroke label "101" after extracting from MiddleLower Zone. 
$[10,16,24,26,27]$. In the present study, Gurmukhi characters are formed by combining the recognized strokes using FSA-based scheme.

\section{Data collection}

In online handwriting recognition system, the creation of standard data for training and evaluation is the first challenge. In this section, we briefly describe the methodology adopted for creating the word level data set for Gurmukhi script.

- Identification of words: This is important to identify a minimal set of words that covers all the defined symbols and can be used as prompts for handwriting data collection. In this connection, we finalized a data set of 126 unique Gurmukhi words, composed of twothree characters.

- Source of data collection: Data have been collected from varied classes of writers. The writers are (i) familiar with Gurmukhi script writing, (ii) students studying in Government schools and Government colleges at diploma/degree level and (iii) professionals, working in Government offices are chosen for the data collection from different places in Punjab (a State of India). A total number of 175 writers, belonging to different age groups, contributed in data collection, shown in table 1.

- Data collection process: An application has been developed and used for collecting the handwriting samples corresponding to the identified data set. A touch-based device, Tablet-PC, has been used for capturing the handwriting samples. Proficiency with the Tablet-PC allows the writer to write in his/her natural handwriting style. Writing on the smooth glass surface using the stylus/digital-pen of the Tablet-PC feels very different from writing on paper. Therefore, a prior training about the writing with Tablet-PC is given to the writer before starting the data collection. Finally, the writer is asked to write the data set of 126 words.

- Annotation: A stroke is the building block in the online handwriting recognition systems as discussed in section 1. Therefore, handwriting information is stored at stroke level. The strokes information such as (i) stroke sequence number, (ii) stroke label, (iii) strokes count and (iv) $x-y$ coordinates values for each point of a stroke is stored in a XML file with respect to a word. In this way, the collected handwritten data are annotated by labelling every stroke with the respective stroke label, depicted in figure 3. We have annotated 65,946 strokes in the present study.

As described in section 3.1, a total of 12 unique stroke classes for Upper Zone and 81 unique stroke classes for MiddleLower Zone have been identified from the collected data. Hence, in order to train the classifiers for both the zones, we have taken 145-170 samples of each identified stroke class. To test the performance of online handwritten Gurmukhi Unicode character recognition system further, a new database was collected from 20 different writers in which each writer is asked to write 41 Gurmukhi consonants 10 times. Hence, in this way, a total of 8200 Gurmukhi characters were collected during this process and further used for testing the proposed system.

\section{Pre-processing and feature extraction}

Generally, the pre-processing operations are applied to remove the variability in the strokes due to the writing styles as well as due to the noise (duplicate points) in the collected data. In the present study, the captured handwritten strokes are partitioned into two zones (i.e., Upper Zone and MiddleLower Zone) [19]. This step helps in reducing the confusion between the similar shape of stroke classes included in Upper Zone and in MiddleLower Zone. After this step, size normalization and centring of the stroke are performed, where the online input stroke is scaled into a fixed size $(300 \times 300)$ of window and moved to the central location. While the strokes are being captured from the Tablet-PC, some of the points of a stroke may be missed due to the limitations of hardware or software and sometimes it may happen due to fast writing of a stroke. In order to retrieve these missing points, Bezier interpolation technique has been used [28]. Thereafter, re-sampling is performed to obtained a constant number of points with uniform distance for all the captured strokes. Therefore, after the interpolation step, the resulting $N$ number of points of each stroke are re-sampled into a fixed size of $64 x$ $y$ coordinates (128 values). The resulting 128 values of $x$ and $y$ coordinates are considered as a feature set in the present study.

The length ( $x-y$ coordinates) of the stroke(s) may vary among different writers. Therefore, to make an efficient stroke classification, we have normalized all the strokes

Table 1. Writers information as age group and gender.

\begin{tabular}{|c|c|c|c|c|c|c|c|}
\hline \multirow[b]{2}{*}{ Writers } & \multicolumn{2}{|c|}{ Age group (10-20) } & \multicolumn{2}{|c|}{ Age group (20-35) } & \multicolumn{2}{|c|}{ Age group (35-50) } & \multirow[b]{2}{*}{ Total } \\
\hline & Male & Female & Male & Female & Male & Female & \\
\hline In training data & 32 & 23 & 37 & 40 & 25 & 18 & 175 \\
\hline In test data & 4 & 3 & 6 & 2 & 3 & 2 & 20 \\
\hline
\end{tabular}


into uniform size, i.e., 64 points, and extracted 128 feature values ( $x$ and $y$ coordinates for each point). Moreover, the efficiency of an online handwritten character recognition system mainly depends on the trained model. For a better recognition accuracy, it is important to identify suitable features. In the present study, we have considered the pre-processed $x-y$ coordinates as features. One can, however, explore the suitability of other features for Gurmukhi script recognition.

\section{Classification}

We have used the LibSVM [29] classifier in our implementation. The key role of SVM classifier in this work is to classify the pre-processed strokes. This classifier takes the arrangement of features according to its standard input file format. The LibSVM classifier can be trained using a feature file consisting of all the classes in order to generate the model file, which can further be used to classify the test data at stroke level. The LibSVM classifier has four different kernels, namely, Linear Kernel, Polynomial Kernel, RBF Kernel and Sigmoid Kernel. It has been seen that effectiveness of the classifier depends on the kernel used, kernel parameters and soft margin or penalty parameter $C$. After carrying out these experiments, we decided to use the RBF Kernel in this work. To measure the robustness of the best predictive model, $K$-fold cross-validation is experimented. The parameters $C$ and $\gamma$ have been considerably varied in order to increase cross-validation accuracy. The model yielding maximum cross-validation accuracy has been used for performance evaluation.

\section{Character formation}

After the recognition of pre-processed strokes using SVM classifier, the recognized strokes are processed further for character generation in post-processing phase. A Gurmukhi character is written using a single stroke, generated easily by mapping the stroke-ID with respective Unicode character. However, when a character is written with more than one stroke, it is necessary to process those strokes in such a way to form a valid Gurmukhi character. As discussed in section 4, a Gurmukhi character can be written with varied number of strokes, use of different combinations of strokes and different sequences of strokes. In this connection, the recognized strokes are processed through several postprocessing steps in order to form a valid Gurmukhi character. After the recognition phase the recognized strokes are stored zone-wise in two lists, namely, UpperZoneList and MiddleLowerZoneList. Each stroke in these lists contains the information on stroke-ID, stroke sequence number and stroke bounding-box knowledge. The two structures, namely, structStrokes and structCharacter, are used to store and process this information for post-processing operations.
The post-processing operations include (i) strokes merging, (ii) extracting lower matras, (iii) re-ordering of recognized strokes' lists and (iv) character formation using FSA, illustrated in figure 6. The recognized strokes from UpperZoneList and MiddleLowerZoneList are processed according to the original input sequence through these postprocessing steps. The strokes from UpperZoneList are mapped to a new list for all the upper matras, referred to as uzlist. For efficient post-processing, the MiddleLowerZoneList is further partitioned into two sub-lists, namely, lzlist-1 and lzlist-2, where lzlist-1 contains all the strokes for Gurmukhi consonants and lzlist-2 contains the lower matras strokes for vowels, and Further, we explore the post-processing operations in detail.

\subsection{Stroke merging}

After the manual verification of handwritten collected data, we observed two strokes: one is vertical-line (') stroke and another one is horizontal-line ('__') stroke, used for writing most of the Gurmukhi characters. These two strokes have different meanings according to their positions with the respective partially written character. During the processing of recognized strokes, if any one of these two strokes occurs, its association (vertical-line association, horizontal-line association) is verified with the processed stroke(s).

8.1a Vertical-line association: The vertical-line stroke has five different types of association according to its position, with the respective partially written character illustrated in figure 7. The Gurmukhi characaters involved in this association are भ, भ, त, Ч, Ч, प, म, घ, थ, घ, ज, ग, ठ, ठ, घ, प्ट and ३. In vertical-line association, the bounding box of the stroke vertical-line is checked for valid association with the bounding box of adjacent processed stroke(s) to form a valid Unicode character. For example some of the Gurmukhi characters such as भ, भ, त, Ч, ษ, य, म, У, щ, घ and ज are usually written by two or more strokes along with a verticalline stroke, where the position of vertical-line stroke is always at the right side to the bounding box of pre-written stroke(s), see figure $7 \mathrm{a}$.

In figure $7 b$, two Gurmukhi characters $\pi$ and $ठ \boldsymbol{r}$ are written similarly as before using two or more strokes along

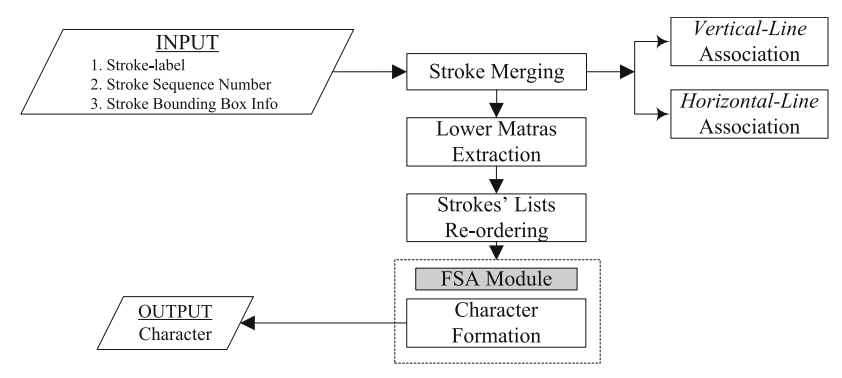

Figure 6. Flow chart of Gurmukhi character formation. 
with a vertical-line stroke. These two characters used the same shapes of strokes, but their lengths of vertical-line strokes are different. Thus, in this case, the length of stroke is the key factor to decide these two different characters. If the length of the vertical-line stroke is greater than a threshold value (i.e., $75 \%$ of the length on $y$-axis of prewritten consonant ' $\nabla$ '), then a single Unicode consonant ' $\mathrm{J}$ ' is formed, shown in figure 8a; otherwise two separate Unicode characters consonant ' $\nabla$ ' and vowel ' $\mathrm{r}$ ' are formed and when these two Unicode characters are rendered in sequence, the character ' $\sigma$ ' ' is formed, demonstrated in figure $8 \mathrm{~b}$.

In this category, those characters in which the verticalline stroke is involved are written at the top-centre position to the bounding box of the post-written stroke, as shown in figure $7 \mathrm{c}$. Similarly, in other categories, those characters come in which the vertical-line stroke is written at the upper-left corner/bottom-right corner to the bounding box of the pre-written stroke(s), seen here in figure $7 \mathrm{~d}$ and e.

8.1b Horizontal-line association: Horizontal-line stroke also plays an important role in Gurmukhi character recognition. This stroke is used for the formation of some of the

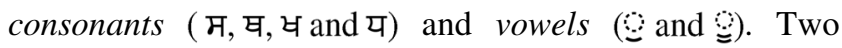
different cases have been found while associating the horizontal-line stroke with the partially written consonant on the basis of its position on $x$-axis and $y$-axis as shown in figure 9. In the first case, the Gurmukhi consonants

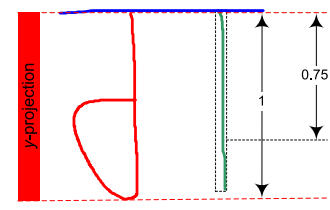

(a)

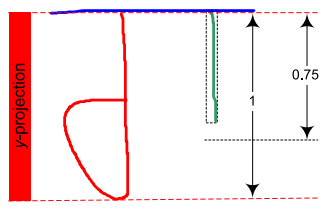

(b)
Figure 8. Decision of vertical line in formation of two different Gurmukhi characters, Jा and Jr. Different colours indicate different strokes.

म, घ and य are formed, when the horizontal-line stroke appears at the upper part of the bounding box of the consonants $\mathcal{H}, \forall$ and $U$, respectively. In the second case, the consonants $\forall$ and घ are formed, when the horizontal-line stroke appears in the middle of bounding box of consonants ४ and य, respectively, by taking the decision of horizontalline stroke on $x$-axis.

\subsection{Lower matras extraction}

In order to process the input stroke sequence more efficiently, stroke labels of three vowels $(3,3$ and have been extracted from MiddleLowerZoneList and moved to lzlist-2 with their sequence number, depicted in figure $5 \mathrm{~b}$. This operation is performed in the Lower Matras Extraction module. This decision is carried out on the basis of $x$ - and

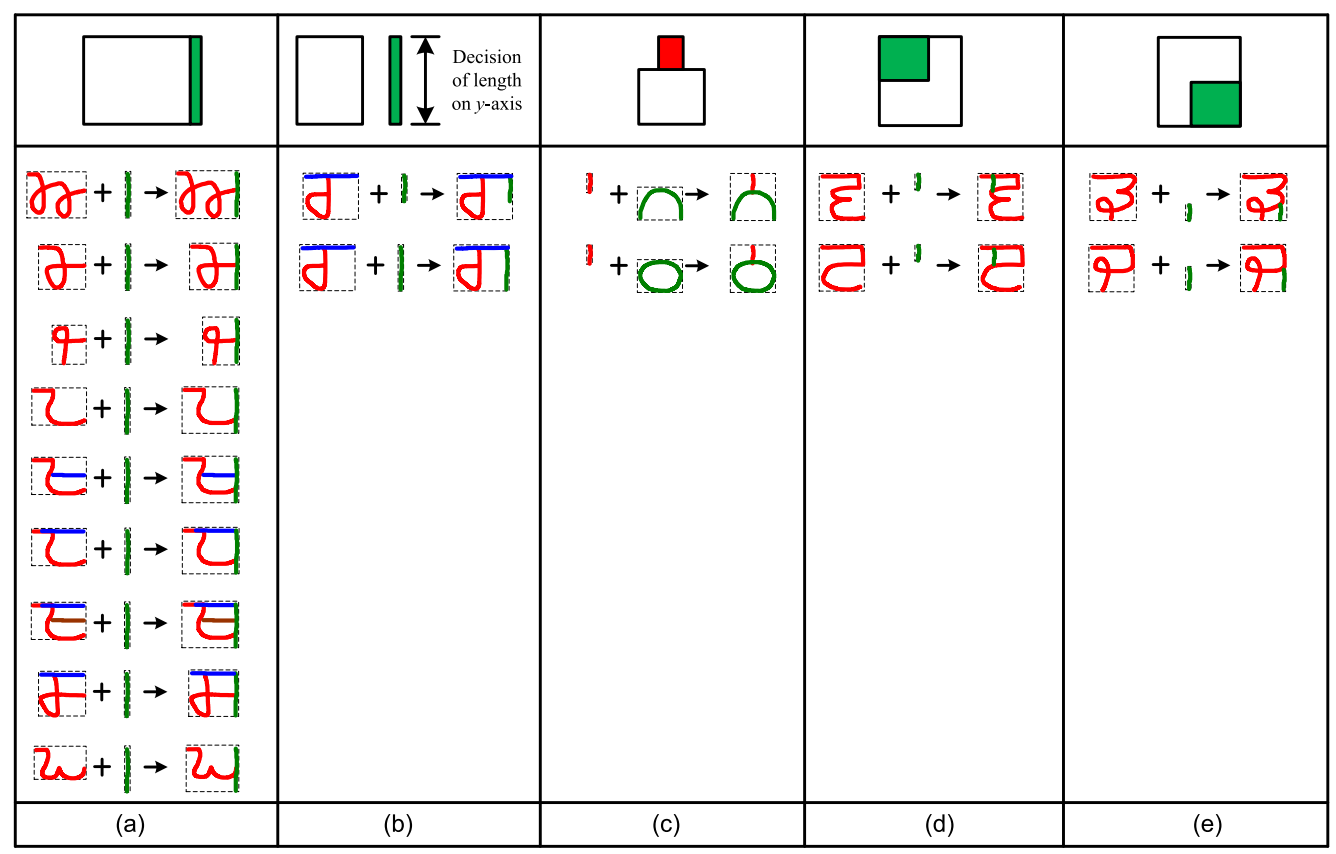

Figure 7. Different cases of vertical-line association with Gurmukhi consonants, where different colours indicate different strokes: (a) examples, where vertical line is written to the right side of the bounding box of the consonant, (b) decision to identify two different characters is carried out on the basis of length of the vertical-line stroke on $y$-axis. Cases where the position of vertical-line stroke is at the top centre, upper left and bottom right are illustrated in $(\mathbf{c}),(\mathbf{d})$ and (e), respectively. 


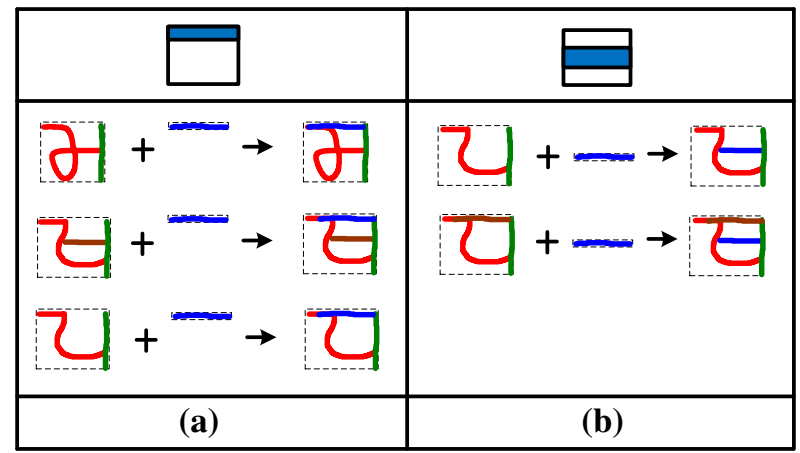

Figure 9. Cases of horizontal-line association with Gurmukhi consonants, where different colours indicate different strokes. The horizontal-line stroke in (a) is written above a consonant and in (b) at the middle to a consonant. $y$-projections of these strokes. Now, the lists uzlist, lzlist-1 and lzlist-2 are added into a single list, alist. This decision of addition is also taken at an intermediate stage when the stroke label of vowel 'f' or 'ी' is encountered.

\subsection{Strokes' lists re-ordering}

The alist consisting of three sub-lists: uzlist, lzlist-1 and lzlist-2 is now ready to form a Unicode character. These strokes' lists need to be rendered in a fixed sequence in order to form a valid Unicode character. In this connection, these strokes' lists are arranged/re-ordered according to the minimum sequence number in each list, as illustrated in figure 10. Finally, these three sub-lists in alist are processed in re-ordered sequence and a valid Unicode character is

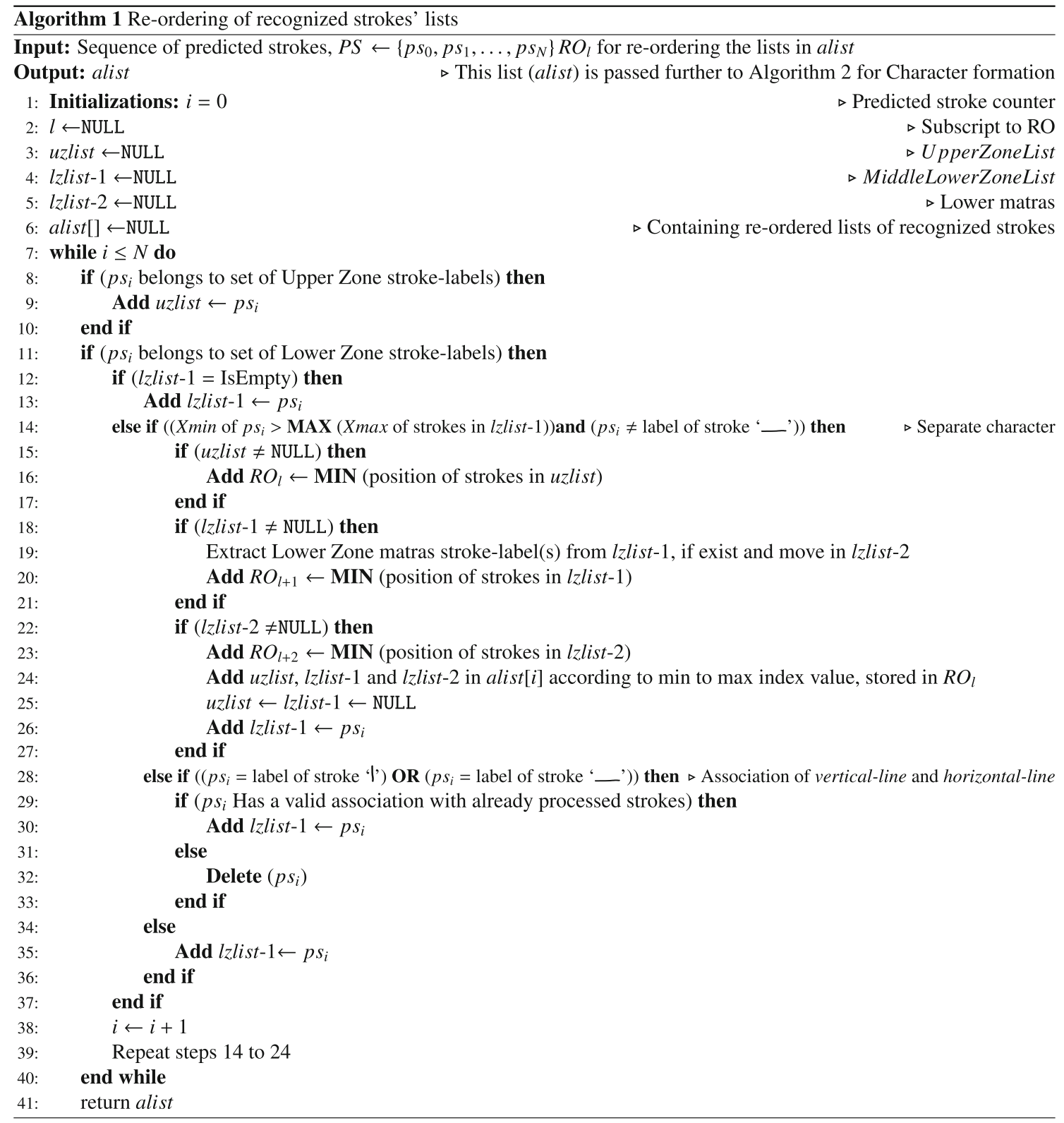




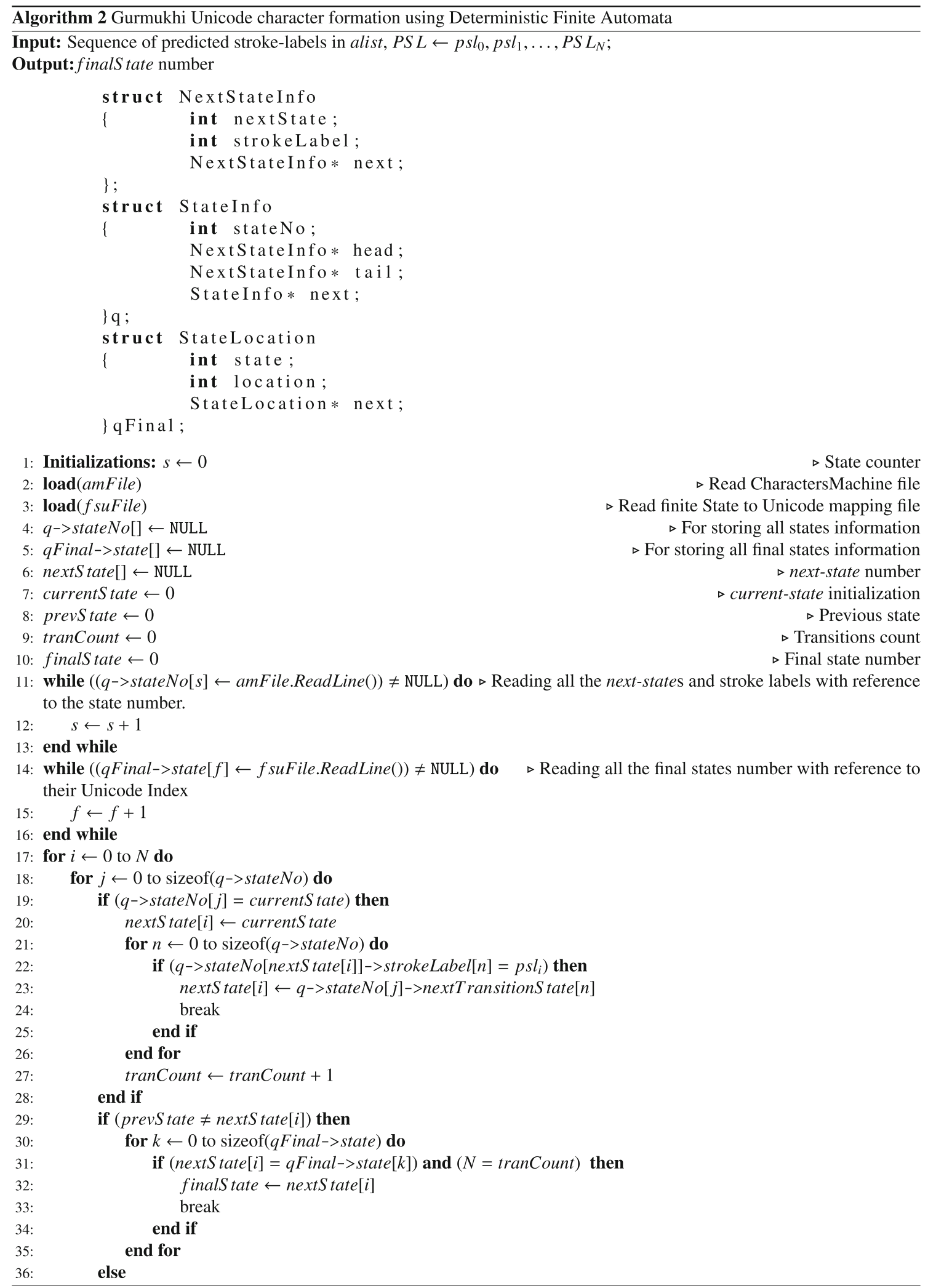



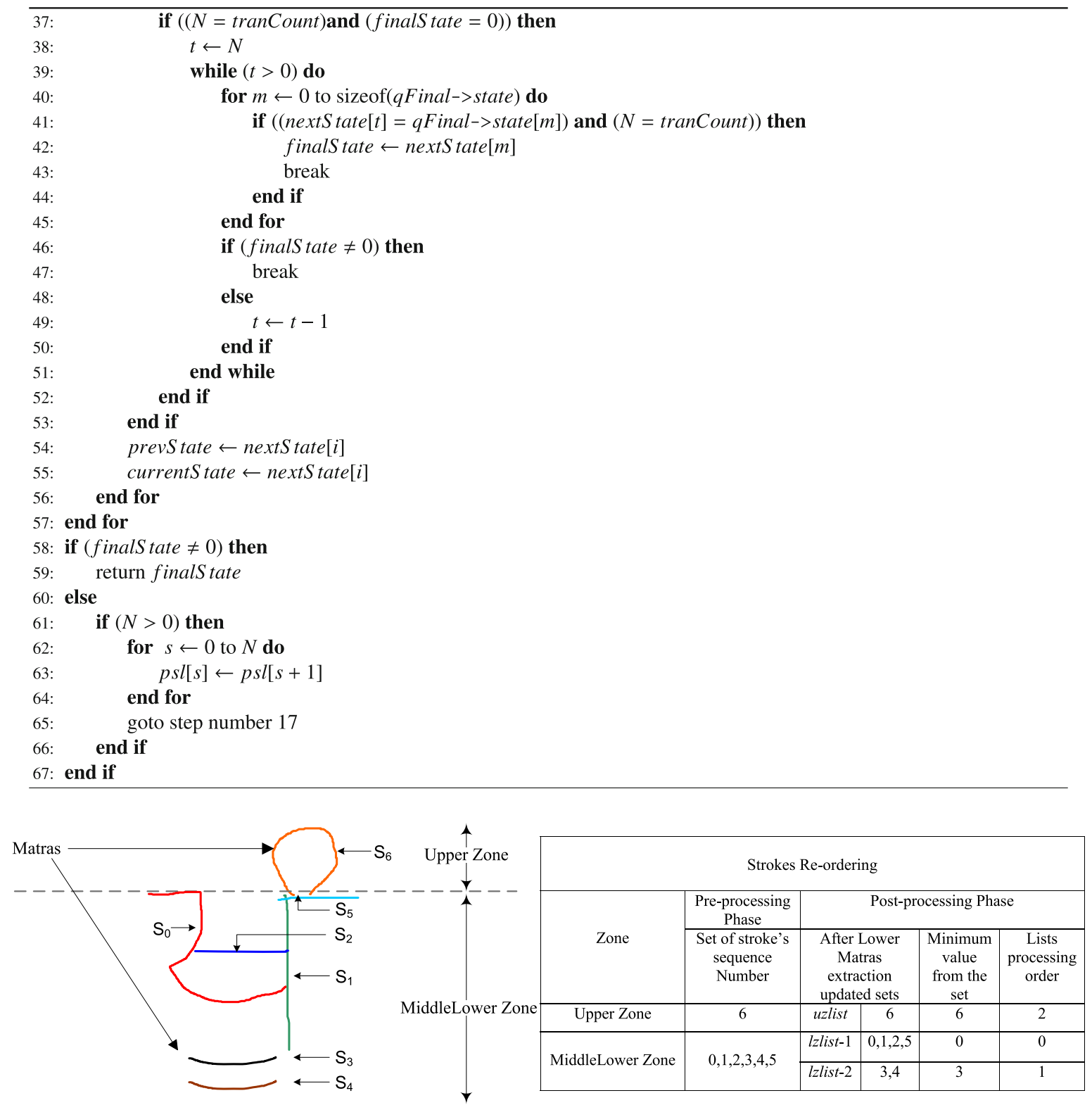

Figure 10. Strokes' lists (uzlist, lzlist-1 and lzlist-2) re-ordering. Different colours indicate different strokes. The zone-wise strokes lists are arranged on the basis of minimum stroke sequence number from the grouped strokes in each list.

formed corresponding to each processed list using FSAbased Gurmukhi character formation algorithm.

\subsection{Character formation using FSA}

The key role of FSA in this work is to form the Gurmukhi Unicode characters from the recognized strokes. A Gurmukhi character can be written using varied number of strokes, different shapes of strokes and different sequences of strokes. Therefore, FSA is implemented in the present work to form a Gurmukhi character by covering all these variations. This section elaborates the character formation process, from the recognized strokes to a formed Gurmukhi character using the proposed FSA-based character formation algorithm. Before employment of FSA, we discuss the basic terminology used in creation of a Finite State Machine (FSM), as an automaton is a study of abstract machines. FSMs are commonly used to organize and represent an execution flow, which is useful for implementation in various applications of AI. The implementation of an FSM begins with the states and transitions it has. The machine has only one state at a time. The state it is in at any given time is called the current state. It can change from one state to another state by triggering an event or a condition, called a transition. A particular FSM is defined by a set of its states, and triggering condition for each transition. Following notations are used to model an FSM. 


$$
\text { Automata }=\left(Q, \Sigma, \delta, q_{0}, F\right)
$$

where $Q$ is a finite set of states, $\Sigma$ is the alphabet (of input symbols), $\delta$ is the transition function, $q_{0} \in Q$ is the initial/ start state and $F \subseteq Q$ is the final state set.

The transition function (Eq. (2), where $\delta$ denotes the transition function, $q_{i}$ and $q_{j}$ are states $\in Q$ and the second parameter (i.e., $a$ ) is an alphabet $\in \Sigma$ ) takes two input parameters, current state of the machine and input symbol a. This function returns next state after processing the input symbol in set $\Sigma$. The next state then becomes the current state when it is processed again with the next symbol in set $\Sigma$. The same process is repeated again and again until the set $\Sigma$ becomes empty.

$$
\delta\left(q_{i}, a\right)=q_{j} .
$$

This state transition function helps in formation of Gurmukhi Unicode character. Initially, the value of the current state is set to zero $\left(q_{i}=0\right)$. When the first predicted stroke label is processed along with the current-state value, the function returns the next state, $q_{j}$. This $q_{j}$ may be either a final state or a non-final state. If $q_{j}$ is a non-final state and there exists any unprocessed predicted stroke label, then it is processed again along with the

\begin{tabular}{|c|c|}
\hline 0 & 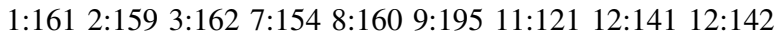 \\
\hline & 13:143 14:144 14:145 17:150 16:182 16:146 18:148 ... \\
\hline 1 & 2:162 4:121 5:163 9:154 2:147 \\
\hline 2 & $9: 1218: 163$ \\
\hline 3 & $\begin{array}{l}2: 161 \quad 6: 163 \quad 55: 207 \text { 7:121 35:174 49:198 50:149 17:146 } \\
44: 36044: 148 \text { 53:202 }\end{array}$ \\
\hline 4 & $9: 162$ \\
\hline 5 & $8: 162$ \\
\hline 6 & $8: 161$ \\
\hline 7 & 9:161 35:174 49:197 55:207 53:202 \\
\hline 8 & $10: 121$ \\
\hline 9 & $10: 163$ \\
\hline 10 & \\
\hline 11 & $\begin{array}{l}4: 161 \quad 7: 162 \quad 9: 159 \quad 12: 141 \quad 12: 142 \quad 13: 143 \quad 19: 189 \quad 26: 157 \\
26 \cdot 15816 \cdot 146 \quad 18 \cdot 148\end{array}$ \\
\hline 12 & $12: 121$ \\
\hline 13 & $13: 121$ \\
\hline 14 & $14: 121 \quad 14: 16214: 147$ \\
\hline
\end{tabular}

Table 2. Gurmukhi character machine file format. next predicted stroke label in the sequence set $\Sigma$. This process is repeated for all the predicted strokes in $\Sigma$ to get a final state. After the completion of this process the final state number is mapped with Unicode Gurmukhi character-set file's index in order to get the respective Unicode character. On the other hand, if the transition function returns a non-final state at the end, the processed set $\Sigma$ is re-submitted to the transition function by eliminating the last predicted stroke label from the sequence set $\Sigma$. This process is followed to search a final state by processing the sub-set of $\Sigma$. The process of eliminating the predicted stroke label starting from the last index in the sequence set $\Sigma$ and resubmission is continued $n-1$ times.

The FSA-based Gurmukhi Unicode character formation algorithm works with three associated files, namely, CharactersMachine file, FinalStateToUnicodeMapping file and UnicodeCharacterSet file. In the CharactersMachine file, all the stroke labels defined in figure 3 are arranged along with their assigned state number (i.e., ' 0 ' or final state). Formation of a Unicode character always starts from the initial state; hence, here all the possible stroke labels, which are usually written as the first stroke for the formation of any Unicode character, are written along with their next state, which is written prior to stroke labels in this file (i.e, next-state: stroke label). Table 2 illustrates the arrangements of states and stroke labels in CharactersMachine file. In the first column of this file, all the final and non-final states have been written and in the front of each state, the movement information to next possible state on the occurrence of stroke label is given. Further, each state has different transitions either to a nonfinal state or final state on the basis of processed predicted stroke label from the sequence of set $\Sigma$. A total of 53 final states have been identified in this implementation. Figure 11 also describes the format of CharacterMachine file.

Similarly, table 3 shows some samples of final states written in FinalStateToUnicodeMapping file. The values of the final states in the second column are mapped with the indices of Unicode characters' indices that are shown in table 4.

\subsection{Validation and working of FSA algorithm}

Validation is possibly the most important step in model building process. Online unconstrained handwritten Gurmukhi characters recognition is a real-time application. Online

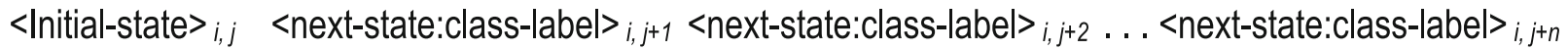

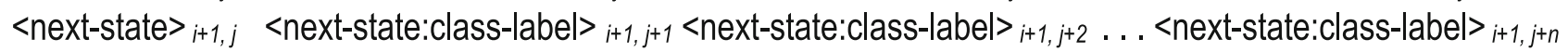$$
\text { . }
$$$$
\cdot
$$

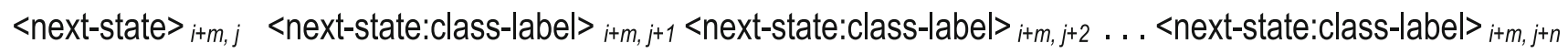

Figure 11. Machine file's structure of Gurmukhi characters, where $i, j, m$ and $n$ are positive integers. 
Table 3. Final state to Unicode index mapping.

\begin{tabular}{lcc}
\hline Sl. no. & Final state & Mapping index \\
\hline 1 & 12 & 1 \\
2 & 14 & 2 \\
3 & 17 & 3 \\
4 & 23 & 4 \\
5 & 25 & 5 \\
6 & 26 & 6 \\
7 & 8 & 7 \\
8 & 28 & 8 \\
9 & 29 & 9 \\
10 & 31 & 10 \\
$\vdots$ & $\vdots$ & $\vdots$ \\
\hline
\end{tabular}

Table 4. Indexing of Unicode Gurmukhi character set.

\begin{tabular}{lc}
\hline Index & Unicode character set \\
\hline 1 & B \\
2 & भ \\
3 & प \\
4 & म \\
5 & ग \\
6 & व \\
7 & ษ \\
8 & ग \\
9 & थ \\
10 & 5 \\
$\vdots$ & $\vdots$ \\
\hline
\end{tabular}

handwritten Gurmukhi Unicode character formation process using Deterministic Finite Automata (DFA) 2 has been explained by testing 8200 characters collected from 20 different writers. This algorithm takes the predicted strokes information (stroke label, stroke sequence number and bounding box information) as an input. The current-state number and stroke label $\left(p s l_{i}\right)$ are passed as parameter $q_{i}$ and ' $a$ ', respectively, to the state transition function, $\delta\left(q_{i}, a\right)=q_{j}$, which returns the next-state number $\left(q_{j}\right)$. Further, this nextstate number $q_{j}$ is processed as prevState along with the next stroke label $p s l_{i+1}$ in sequence set $\Sigma$, to get the next-state number $q_{j+1}$. Similarly, this process is repeated for all the stroke labels in $\Sigma$ with the updated value of next state, which is produced by the previous iteration. Finally, after processing all the strokes from $\Sigma$, the last value prevState in the next state is checked as to whether it is a final state number or not. If this prevState value is matched with the final state value, then the corresponding Gurmukhi Unicode character is returned by mapping the final state value to the index value of the Gurmukhi Unicode character set, defined in table 4. On the other side, the line numbers [37-52] and [58-67] are executed only if the prevState value does not match with the final state value. This snippet of code searches the final state by matching the traversed states in next state in reverse order, with all the final states described in table 3 . If found, it returns a respective Unicode character; otherwise, this strokes set $\Sigma$ is processed again to this state transition function after eliminating the first stroke label from the sequence set $\Sigma$ in order to get the Gurmukhi Unicode character output, similar in shape to the input. Figure 12a shows the formation process of Gurmukhi Unicode characters $4, \forall, ~ Y$ and घ using an FSA-based finite machine. A finite set of stroke labels are defined in this figure to form these Gurmukhi Unicode characters. Hence, the representation model for this machine is deterministic. Following notations have been used in the implementation of this FSM.

$$
\begin{aligned}
Q & =\mathrm{S} 0, \mathrm{~S} 1, \mathrm{~S} 2, \ldots \mathrm{S} 11, \\
\Sigma & =121,159,160,161,162,163,195, \\
q_{0} & =\mathrm{S} 0, \\
F & =\mathrm{S} 2, \mathrm{~S} 8, \mathrm{~S} 9, \mathrm{~S} 10 .
\end{aligned}
$$

In this FSM, we have covered all the possible combinations of stroke labels that help in the formation of these Unicode characters. For example ' 2 ' can be formed using two different ways from the initial state S0: (i) with stroke label '159' and (ii) with stroke labels ' 161 ' and '162'. Gurmukhi character ' $\mathrm{y}$ ' is formed in six different ways. Similarly ' ' is formed in seven different ways. Finally, the character ' $\mathbf{}$ ' has totally 13 variations. State transition, from the initial state to the final state on the occurrence of stroke labels, has been discussed in the state transition table (table 5). The states in bold text are the final states (i.e., S2, S8, S9 and S10). With the same idea, we have implemented totally 35 machines that are used for the formation of 53 Gurmukhi Unicode characters. The FSM structure helps in reducing both memory and execution time during the formation of Gurmukhi Unicode characters. Similarly, figure $12 \mathrm{~b}$ illustrates the formation of Gurmukhi lower matra Unicode character ' mukhi upper matra Unicode character ' $\gg$ ' in three different ways.

\section{Experimentation and comparative study}

This section briefly describes about the experiments carried out for training the zone-wise classifiers, testing the performance of the proposed recognition system, comparison of the present work to state-of-the-art and error analysis for online handwritten character recognition.

\subsection{Training the classifier}

To implement the online handwritten Gurmukhi character recognition system, two zone-wise classifiers 


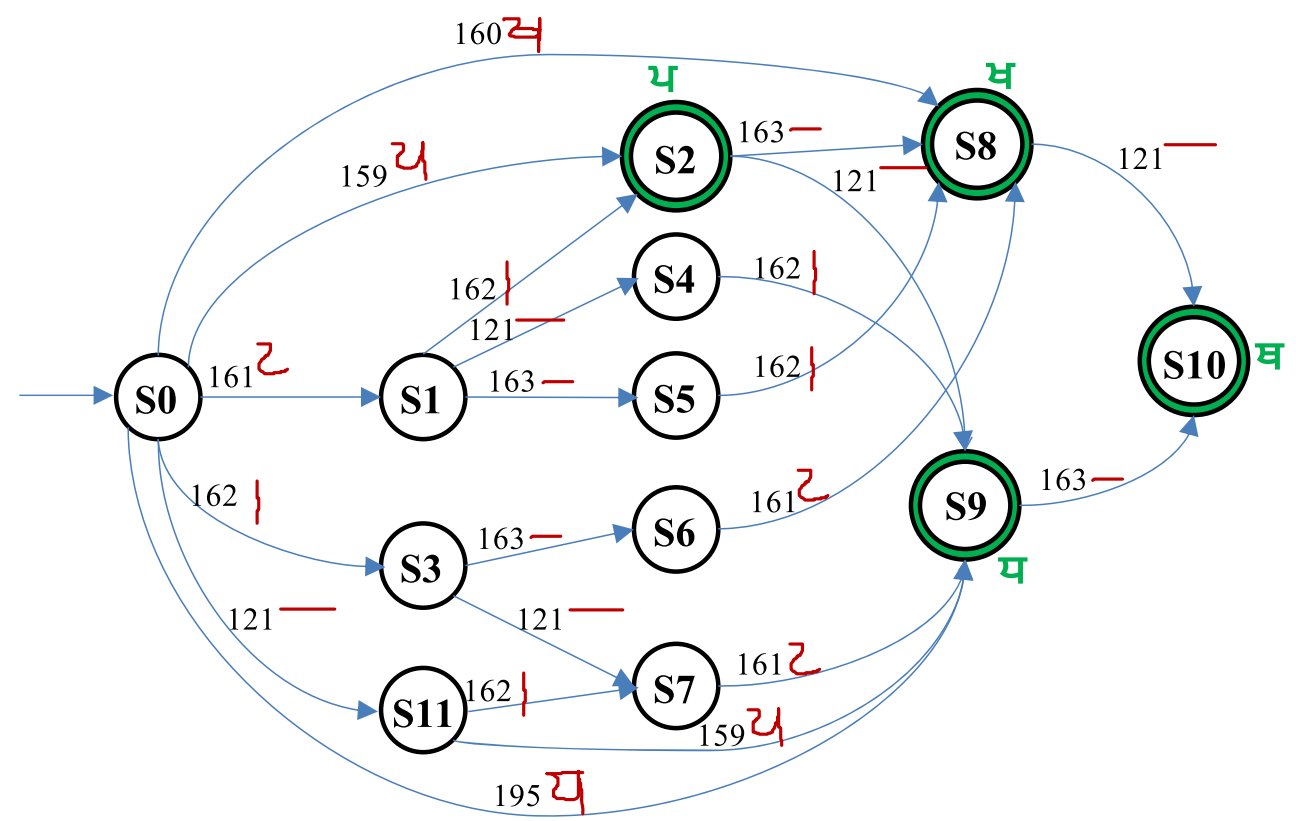

(a) Illustration of all the possible variations to form the Gurmukhi characters $\mathrm{Y}, \mathrm{Y}, \mathrm{Z}$ and $\mathrm{B}$ from strokes.

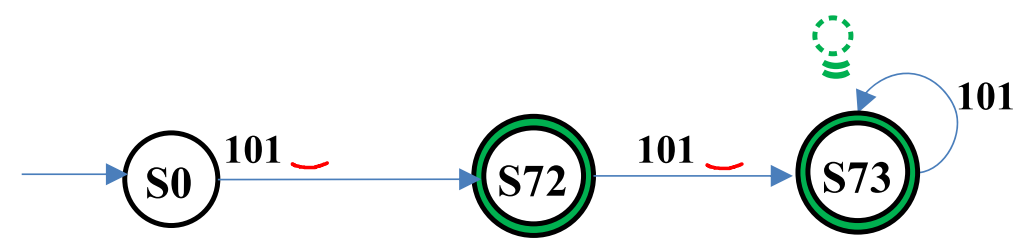

(b) Formation of lower matra, $\stackrel{⿹}{=}$.

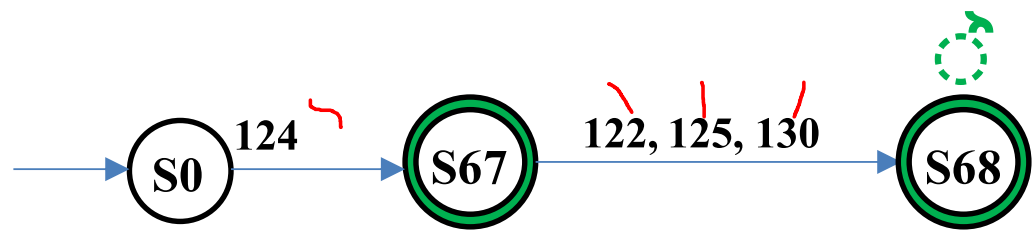

(c) Formation of upper matra, 'x, on the occurrence of different classes of stroke.

Figure 12. Examples of Gurmukhi Unicode characters formation using Finite State Automata's machines, where a single circle represents a non-final state and double circles represent the final state.

UpperZone-Classifier and MiddleLowerZoneClassifier are trained using SVM classifier. The pre-processed strokes in their respective zones are classified with these two zonewise classifiers. A total of 1,872 samples of stroke classes are considered for training the UpperZoneClassifier and 13,197 samples of stroke classes are considered for MiddleLowerZoneClassifier. In order to make a better training model, a few experiments have been carried out on these data sets, by tuning the penalty parameter $(C)$ and learning rate $(\gamma)$ of RBF kernel on different folds. The value of $\log _{2}(C)$ and $\log _{2}(\gamma)$ varies over the interval ( $\left.-5(1) 15\right)$ and $(3(-1)-15)$, respectively. Table 6 contains the values of parameters and cross-validation accuracy achieved by the proposed system for two zones. The highest cross-validation accuracy of $99.08 \% \quad\left(k=7, \quad \log _{2}(C)=6\right.$, $\left.\log _{2}(\gamma)=-7\right)$ is achieved for UpperZoneClassifer and 97.38\% $\left(k=8, \log _{2}(C)=7, \log _{2}(\gamma)=-9\right)$ for MiddleLowerZoneClassifier. These two classifiers are further used for predicting the pre-processed strokes in Upper Zone and MiddleLower Zone, respectively.

\subsection{Testing}

For testing the performance of the proposed character recognition approach, a data set of 8,200 samples of online handwritten Gurmukhi Unicode characters, written by 20 new writers, has been considered to test the utility of the proposed character recognition system. A "Dell-latitude XT-3" Tablet-PC device was used to collect these data. Initially, the online handwritten character's stroke(s) was 
Table 5. State transition table for Gurmukhi Unicode characters.

\begin{tabular}{lccccccc}
\hline & \multicolumn{7}{c}{ Input symbols } \\
\cline { 2 - 8 } Transition & 121 & 159 & 160 & 161 & 162 & 163 & 195 \\
\hline S0 & S11 & S2 & S8 & S1 & S3 & - & S9 \\
S1 & S4 & - & - & - & S2 & S5 & - \\
S2 & S9 & - & - & - & - & S8 & - \\
S3 & S7 & - & - & - & - & S6 & - \\
S4 & - & - & - & - & S9 & - & - \\
S5 & - & - & - & - & S8 & - & - \\
S6 & - & - & - & S8 & - & - & - \\
S7 & - & - & - & S9 & - & - & - \\
S8 & S10 & - & - & - & - & - & - \\
S9 & - & - & - & - & - & S10 & - \\
S10 & - & - & - & - & - & - & - \\
S11 & - & S9 & - & - & S7 & - & - \\
\hline
\end{tabular}

(were) processed through the pre-processing steps in order to get the feature vector of $64 x-y$ co-ordinates values against each processed stroke. After this, the pre-processed strokes are processed further for the recognition with the respective zone classifier. The zone-wise classifiers produce a unique stroke identification number (i.e., stroke label) corresponding to the processed pre-processed stroke. This process is repeated for all the pre-processed strokes. Finally, the recognized strokes are processed for character formation with some additional information about each strokes, such as (i) stroke label, (ii) stroke sequence number and (iii) stroke bounding box information. The proposed Gurmukhi character formation approach forms a valid Unicode character after processing the recognized strokes.

\subsection{Comparison with state-of-the-art}

Table 7 illustrates the character-wise result obtained for 41 basic Gurmukhi characters, including 6 additional modified

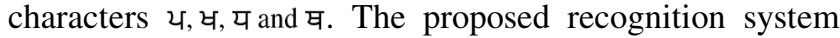
achieved an accuracy of $97.3 \%$ for these 41 Gurmukhi characters. The average character recognition time in milliseconds is also shown in the table. The character recognition time is computed as total time taken by the recognition system from submission time of input strokes to the final character generation.

The proposed algorithm achieved a good amount of accuracy of $95.8 \%$ for characters $\cong$, which is better than the achieved accuracy of $88.5 \%$ using the proposed work by Verma and Sharma [12]. The proposed system also overcomes the limitations of the work proposed by Kumar and Sharma [10] as follows.

(i) The problem related to dealing with unknown sequence of strokes and characters has been resolved in the present work. It is fixed by restricting the transition for the unknown stroke. Figure 13 illustrates the example of formation of the Gurmukhi character $\hat{\lambda}$, from input sequence of stroke labels "148", "146" and "147". The stroke label "147" is an extra/unknown stroke in the current stroke sequence set. Therefore, no transition will take place on processing of stroke label "147". After processing first two stroke labels, the current state will be a final state (i.e., double circled) and from this state there is a self-loop for stroke label "147". Hence, the output

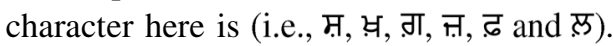

(ii) The problem of writing the same stroke more than once and problem of occurrence of the noisy stroke at the end or at the beginning in a stroke sequence are also resolved by restricting the transition to the same state on the occurrence of such stroke label, as depicted in figure 13.

The study on online handwritten Gurmukhi script recognition in the recent past is summarized in table 8 , where the authors have reported character recognition accuracy using

Table 6. Values of parameters and stroke-level accuracy achieved for two zones.

\begin{tabular}{|c|c|c|c|c|c|c|}
\hline Zone & Number of classes & Number of samples & Value of $k$ in $k$-fold cross-validation & $\log _{2}(C)$ & $\log _{2}(\gamma)$ & Accuracy $(\%)$ \\
\hline \multirow[t]{7}{*}{ Upper } & \multirow[t]{7}{*}{12} & \multirow[t]{7}{*}{1872} & 3 & 4 & -7 & 98.67 \\
\hline & & & 4 & 6 & -7 & 98.58 \\
\hline & & & 5 & 6 & -7 & 98.83 \\
\hline & & & 6 & 6 & -7 & 99.00 \\
\hline & & & 7 & 6 & -7 & 99.08 \\
\hline & & & 8 & 4 & -7 & 98.91 \\
\hline & & & 9 & 4 & -7 & 99.00 \\
\hline \multirow[t]{7}{*}{ MiddleLower } & \multirow[t]{7}{*}{81} & \multirow[t]{7}{*}{13197} & 3 & 4 & -9 & 96.96 \\
\hline & & & 4 & 5 & -9 & 97.11 \\
\hline & & & 5 & 7 & -11 & 97.21 \\
\hline & & & 6 & 7 & -11 & 97.31 \\
\hline & & & 7 & 7 & -9 & 97.31 \\
\hline & & & 8 & 7 & -9 & 97.38 \\
\hline & & & 9 & 4 & -11 & 97.15 \\
\hline
\end{tabular}


Table 7. Character-wise recognition accuracy.

\begin{tabular}{|c|c|c|c|c|c|}
\hline $\begin{array}{l}\text { Gurmukhi } \\
\text { characters }\end{array}$ & $\begin{array}{c}\text { Recognition } \\
\text { accuracy }\end{array}$ & $\begin{array}{l}\text { Confusing } \\
\text { characters }\end{array}$ & $\begin{array}{l}\text { Gurmukhi } \\
\text { Characters }\end{array}$ & $\begin{array}{c}\text { Recognition } \\
\text { Accuracy }\end{array}$ & $\begin{array}{l}\text { Confusing } \\
\text { Characters }\end{array}$ \\
\hline 8 & 100.0 & & 3 & 97.5 & उ \\
\hline n & 100.0 & & घ & 97.5 & ધ \\
\hline प्ट & 94.0 & $\varepsilon$, हृ & E & 100.0 & \\
\hline म & 96.0 & भ & य & 98.5 & પ \\
\hline J & 96.5 & ठ & চ & 96.5 & ट \\
\hline व & 100.0 & & ૫ & 98.5 & य \\
\hline ધ & 98.5 & घ & ढ & 100.0 & \\
\hline गा & 95.5 & ग', ग & घ & 96.5 & हा \\
\hline wr & 96.5 & ૫ & उ & 98.5 & 3 \\
\hline 5 & 100.0 & & भ & 96.5 & म \\
\hline$\theta$ & 100.0 & & ज & 97.5 & य \\
\hline ह & 100.0 & & J & 99.0 & J \\
\hline त & 98.5 & व & ম & 95.0 & प्ट \\
\hline ₹ & 94.5 & वॅ & द & 100.0 & \\
\hline घ & 93.5 & ाह्द, प्ट & इ & 96.5 & इ \\
\hline ट & 100.0 & & म & 93.0 & म \\
\hline б & 98.5 & ट & y & 92.5 & $\underline{y}$ \\
\hline उ & 100.0 & & गा & 91.5 & ग \\
\hline द & 100.0 & & त़ & 93.5 & छु, व \\
\hline$\varepsilon$ & 95.5 & प्र & ढ & 97.5 & ढ \\
\hline & & & पू & 94.5 & एू, प्ट \\
\hline \multicolumn{4}{|c|}{ Average recognition rate $(\%)$} & 97.3 & \\
\hline \multicolumn{4}{|c|}{ Average recognition time (in $\mathrm{ms}$ ) (\%) } & 27.9 & \\
\hline
\end{tabular}

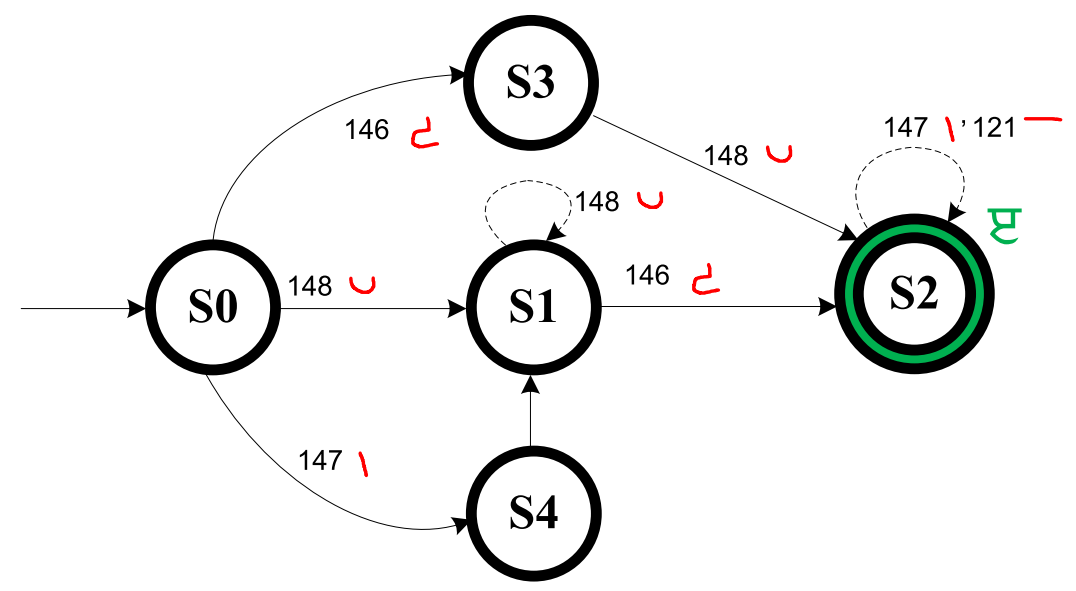

Figure 13. Example of handling the occurrence of same stroke more than once and extra stroke(s).

varied number of stroke classes and character formation approaches considered. Sharma et al [21] have achieved $90.1 \%$ accuracy at character level using the elastic matching scheme. Later on they used HMM classifier in their work and achieved $91.9 \%$ accuracy. Kumar and Sharma [10] used the rule base character formation strategy in postprocessing and achieved $95.6 \%$ accuracy. Verma and
Sharma [12] proposed the character recognition system using voting-based classifiers and employed $96.7 \%$ accuracy. In their work, a single classifier is used to classify the captured strokes. Due to this reason, their system encountered the issues related to strokes misclassification. However, in the present study, the captured strokes are classified into two zones to overcome the problem of strokes 
Table 8. Comparison analysis with earlier approaches for Gurmukhi character recognition.

\begin{tabular}{lccc}
\hline References & Stroke classes & Classification techniques & Character accuracy $(\%)$ \\
\hline Sharma et al [20] & 40 & Elastic matching & 90.1 \\
Sharma et al [22] & 40 & HMM & 91.9 \\
Kumar and Sharma [10] & 114 & SVM, Rule based & 95.6 \\
Kumar et al [11] & 114 & SVM, Rule based & 93.3 \\
Verma and Sharma [24] & 102 & SVM & 92.2 \\
Verma and Sharma [12] & 74 & SVM, HMM & 96.7 \\
Current work & 93 & SVM, FSA based & 97.3 \\
\hline
\end{tabular}

\begin{tabular}{|c|c|c|}
\hline Character & $\begin{array}{c}\text { Standard } \\
\text { writing order }\end{array}$ & $\begin{array}{c}\text { Observed order } \\
\text { and output }\end{array}$ \\
\hline fि & ठ ि & ि+ ठ $\rightarrow$ िठ \\
\hline वि & व ि & िव $\rightarrow$ िव \\
\hline मे & म+े + & म+े क मे \\
\hline
\end{tabular}

Figure 14. Standard and observed rendering order of consonant and $\operatorname{vowel}(s)$.

misclassification. Moreover, an efficient FSA-based Gurmukhi character formation algorithm has been employed in post-processing, which produces a higher recognition accuracy of $97.3 \%$. As observed, the character recognition accuracy achieved using FSA is more than those from the other earlier proposed schemes.

\subsection{Error analysis}

Though the proposed FSA-based formation of online handwritten Gurmukhi character performs sufficiently well, it still has some errors. The errors are mostly of two types.

(i) Misclassification of strokes: Incorrect Gurmukhi character formation due to processing of one or more misclassified stroke(s). The present system achieved an average recognition accuracy of $93.8 \%$ for six additional modified characters गा, ₹, ह and घ. The reason for this low recognition accuracy is misclassification of strokes, such as stroke labels "102" ' $\mathrm{C}$ ' and "222" ' $\mathrm{C}$ ', confused with " 101 "

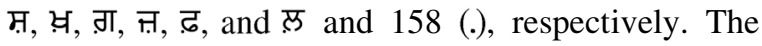
main issue associated with writing the vowels $(\mathcal{P})$ is that mostly very few number of points (i.e., 5-10 points) are captured while writing them, which are insufficient to construct the feature vector of size 64 $x-y$ points during pre-processing.

(ii) Incorrect rendering order of consonant and vowel(s): The order in which consonant and vowel(s) constituting a single character are written varies across individuals. Some variations observed during the testing phase are shown in figure 14 .

\section{Conclusions and future directions}

This paper presents an online handwritten Gurmukhi character recognition using FSA theory. The issues that are probably encountered in the formation of handwritten characters have been discussed in this study. A total of 93 stroke classes have been identified for Gurmukhi characterset formation. Initially, the online handwritten character is processed through pre-processing steps: normalization, duplicate points removal, interpolation and re-sampling. Two SVM-based classification models, namely, UpperZoneClassifier and MiddleLowerZoneClassifier, have been used for stroke classification in their respective zones. In post-processing phase the stroke merging, lower matras extraction, re-ordering the strokes lists and character formation steps are carried out in order to form a valid Unicode character. In online handwritten Gurmukhi character formation, we have covered all the variations used to write a Gurmuki character by different individuals, using the proposed FSA-based character formation algorithm. The proposed work presents a higher recognition accuracy of 97.3\% for Gurmukhi Unicode character recognition using FSA-based theory. It is observed that the character recognition accuracy achieved using FSA is more than those from the other earlier proposed schemes, shown in table 8 .

Our future attempts will be towards improving the stroke classification by adding different classifiers based on deep learning, enhancing the recognition performance at word level, development of an android-based recognition system and incorporating a language model for improving the postprocessing at word-formation level.

\section{Acknowledgements}

We take this opportunity to extend our special thanks to Technology Development for Indian Languages (TDIL), DeitY, MoCIT and Government of India for sponsoring this research work. 


\section{References}

[1] Liu C L, Jaeger S and Nakagawa M 2004 Online recognition of Chinese characters: the state-of-the-art. IEEE Trans. Pattern Anal. Mach. Intell. 26(2): 198-213

[2] Jäger S, Liu C L and Nakagawa M 2003 The state of the art in Japanese online handwriting recognition compared to techniques in western handwriting recognition. Docum. Anal. Recognit. 6(2): 75-88

[3] Jung K C, Yoon S M and Kim H J 2000 Continuous HMM applied to quantization of on-line Korean character spaces. $J$. Pattern Recognit. Lett. 21(4): 303-310

[4] Sternby J, Morwing J, Andersson J and Friberg C 2009 Online Arabic handwriting recognition with templates. J. Pattern Recognit. 42(12): 3278-3286

[5] Bhattacharya N and Pal U 2012 Stroke segmentation and recognition from Bangla online handwritten text. In: Proceedings of the International Conference on Frontiers in Handwriting Recognition (ICFHR), pp. 740-745

[6] Bharath A and Madhvanath S 2012 HMM-based lexicondriven and lexicon-free word recognition for online handwritten Indic scripts. IEEE Trans. Pattern Anal. Mach. Intell. 34(4): 670-682

[7] Biswas C, Bhattacharya U and Parui S K 2012 HMM based online handwritten Bangla character recognition using Dirichlet distributions. In: Proceedings of the International Conference on Frontiers in Handwriting Recognition (ICFHR), pp. 600-605

[8] Sundaram S and Ramakrishan A G 2011 Lexicon-free, novel segmentation of online handwritten Indic words. In: Proceedings of the International Conference on Document Analysis and Recognition, pp. 1175-1179

[9] Prasad M M, Sukumar M and Ramakrishnan A G 2009 Divide and conquer technique in online handwritten Kannada character recognition. In: Proceedings of the International Workshop on Multilingual OCR, p. 11

[10] Kumar R and Sharma R K 2013 An efficient post processing algorithm for online handwriting Gurmukhi character recognition using set theory. Int. J. Pattern Recognit. Artif. Intell. 27(04): 1353002(1-16)

[11] Kumar R, Sharma R K and Sharma A 2015 Recognition of multi-stroke based online handwritten Gurmukhi aksharas. In: Proc. Natl. Acad. Sci. India Sect. A Phys. Sci. 85(1): 159-168

[12] Verma K and Sharma R K 2017 Comparison of HMM-and SVM-based stroke classifiers for Gurmukhi script. Neural Comput. Appl. 28(1): 51-63

[13] Tappert C C, Suen C Y and Wakahara T 1990 The state of the art in online handwriting recognition. IEEE Trans. Pattern Anal. Mach. Intell. 12(8): 787-808

[14] Gao J, Zhu B and Nakagawa M 2014 Building compact recognizer with recognition rate maintained for on-line handwritten Japanese text recognition. J. Pattern Recognit. Lett. 35: 169-177
[15] Aparna K H, Subramanian V, Kasirajan M, Prakash G V, Chakravarthy V S and Madhvanath S 2004 Online handwriting recognition for Tamil. In: Proceedings of the 9th International Workshop on Frontiers in Handwriting Recognition, pp. 438-443

[16] Swethalakshmi H, Jayaraman A, Chakravarthy V S and Sekhar C C 2006 Online handwritten character recognition of Devanagari and Telugu Characters using support vector machines. In: Proceedings of the 10th International Workshop on Frontiers in Handwriting Recognition, Suvisoft

[17] Rampalli R and Ramakrishnan A G 2011 Fusion of complementary online and offline strategies for recognition of handwritten Kannada characters. J. Univers. Comput. Sci. 17(1): 81-93

[18] Roy K, Bandhopadhyay A and Mondal R 2012 Strokedatabase design for online handwriting recognition in Bangla. Int. J. Mod. Eng. Res. 2(4): 2534-2540

[19] Samanta O, Bhattacharya U and Parui S K 2014 Smoothing of HMM parameters for efficient recognition of online handwriting. J. Pattern Recognit. 47(11): 3614-3629

[20] Sharma A, Kumar R and Sharma R K 2008 Online handwritten Gurmukhi character recognition using elastic matching. In: Proceedings of the IEEE Congress on Image and Signal Processing, Hainan, China, pp. 391-396

[21] Sharma A, Kumar R and Sharma R K 2009 Rearrangement of recognized strokes in online handwritten Gurmukhi words recognition. In: Proceedings of the 10th International Conference on Document Analysis and Recognition, Barcelona, Spain, pp. 1241-1245

[22] Sharma A, Kumar R and Sharma R K 2010 HMM-based online handwritten Gurmukhi character recognition. Int. J. Mach. Graph. Vis. 19(4): 439-449

[23] Singh G and Sachan M 2015 A framework of online handwritten Gurmukhi script recognition. Int. J. Comput. Sci. Technol. 6: 52-56

[24] Verma K and Sharma R K 2015 Performance analysis of zone based features for online handwritten Gurmukhi script recognition using Support Vector Machine. In: Progress in Systems Engineering, Springer, pp. 747-753

[25] Bahri H 1982 Teach yourself Panjabi. Patiala: Publication Bureau, Panjabi University

[26] Chen J W and Lee S Y 1996 On-line handwriting recognition of Chinese characters via a rule-based approach. In: Proceedings of the 13th International Conference on Pattern Recognition, vol. 3, pp. 220-224

[27] Jayaraman A, Sekhar C C and Chakravarthy V S 2007 Modular approach to recognition of strokes in Telugu script. In: Proceedings of the 9th International Conference on Document Analysis and Recognition, vol. 1, pp. 501-505

[28] Sharma A, Sharma R K and Kumar R 2009 Online handwritten Gurmukhi character recognition. $\mathrm{PhD}$ Thesis, Thapar University, Patiala, India

[29] Chang C C and Lin C J 2011 LIBSVM: a library for support vector machines. ACM Trans. Intell. Syst. Technol. 2(3): 27 